\title{
Constraining How Star Formation Proceeds: Surveys in the Sub-mm and FIR
}

\author{
Doug Johnstone ${ }^{1,2}$ \\ ${ }^{1}$ National Research Council Canada, Herzberg Institute of Astrophysics, \\ 5071 West Saanich Rd, Victoria, BC, V9E 2E7, Canada \\ email: doug.johstone@nnrc-cnrc.gc.ca \\ ${ }^{2}$ Department of Physics \& Astronomy, University of Victoria, Victoria, BC, V8P 1A1, Canada
}

\begin{abstract}
Coordinated multi-wavelength surveys of molecular clouds are providing strong constraints on the physical conditions within low-mass star-forming regions. In this manner, Perseus and Ophiuchus have been exceptional laboratories for testing the earliest phases of star formation. Highlights of these results are: (1) dense cores form only in high column density regions, (2) dense cores contain only a few percent of the cloud mass, (3) the mass distribution of the dense cores is similar to the IMF, (4) the more massive cores are most likely to contain embedded protostars, and (5) the kinematics of the dense cores and the bulk gas show significant coupling.
\end{abstract}

\section{Core Locations Within Molecular Clouds}

Large samples of pre- and proto-stellar cores within nearby molecular clouds have been investigated primarily through (sub)millimeter continuum observations with singledish telescopes, which preferentially reveal small and dense regions of dust emission and therefore are ideal for locating the cores. Two such surveys, (Johnstone et al. (2004) and Kirk et al. (2006)), taken with SCUBA at the JCMT, have covered significant portions of Ophiuchus and Perseus respectively.

Comparison between the core locations and the bulk material in the clouds, as measured by near IR extinction [see Lombardi \& Alves (2001) for the technique], reveals that the cores are biased toward the highest column density regions (measured in $A_{v}$ of extinction) within their individual clouds. Despite the fact that most of the mass of the molecular cloud is found at low column density, few cores are found in these extended regions. In Ophiuchus the cores are almost exclusively found above $A_{v}=15$ Johnstone et al. (2004) while the mean $A_{v}$ is 4 . In Perseus the cores are almost exclusively found above $A_{v}=5$ Kirk et al. (2006) while the mean $A_{v}$ is only 2 . Interestingly, the Perseus cores appear slightly offset from the column density peaks within the cloud, perhaps evidence for triggering events playing an important role in producing cores and protostars.

The mass of the cloud locked up within the cores is minimal, as might be expected given the low star formation efficiency expected for clouds. For both Perseus and Ophiuchus, the total mass in cores is only a few percent of the entire cloud mass. Even in the higher column density zones, the cores only account for ten to twenty percent of the cloud mass Johnstone et al. (2004), Kirk et al. (2006). This is an important point to recognize: the cores appear to be a separate, insignificant, component of the cloud and their physical properties should be considered distinct from those of the bulk cloud.

\section{Core Properties}

Considering the cores as a distinct sample reveals that the core mass function has a similar appearance to that of stars (see Ward-Thompson et al. (2007) and references 
therein); the majority of cores and the majority of the mass in the cores is found in the lower mass cores - like for stars. Whether this relationship is evidence for the masses of stars being pre-selected at the core stage is debatable but the distribution does provide a starting point for comparison between observations and simulations Hatchell \& Fuller (2008), Swift \& Williams (2008).

Collating mid-IR observations of star-forming regions taken by Spitzer Evans et al. (2003) with the submillimeter core maps allows for a robust determination of which cores harbor proto-stellar sources Jørgensen et al. (2007), Jørgensen et al. (2008). Detailed analysis shows that the proto-stellar cores are more massive and more likely to be highly concentrated than the starless cores, as might be expected if the massive cores are more likely to collapse under their own weight. As well, mid-IR detections, indicating a heating source within the proto-stellar cores, are strongly biased toward the center of each submillimeter core, implying that the proto-star is kinematically coupled to the core Jørgensen et al. (2007).

\section{Core Kinematics}

The cores themselves appear kinematically coupled to the bulk cloud, as noted by Kirk et al. (2007) in an analysis of $\mathrm{N}_{2} \mathrm{H}^{+}(1-0)$ and $\mathrm{C}^{18} \mathrm{O}(2-1)$ line profiles. The $\mathrm{N}_{2} \mathrm{H}^{+}$ line widths are dominated by thermal motions, especially for the starless core sample [this is also seen in a complementary $\mathrm{NH}_{3}(1,1)$ dataset Rosolowsky et al. (2008)]. The $\mathrm{C}^{18} \mathrm{O}$ gas, however, preferentially probes the bulk gas surrounding the core and reveals a much wider line width, consistent with the notion that non-thermal motions dominate on larger scales within molecular clouds. Interestingly, the line centroids of the $\mathrm{C}^{18} \mathrm{O}$ and $\mathrm{N}_{2} \mathrm{H}^{+}$observations are typically offset by less than the sound speed, suggesting that the non-thermal motions in the cloud do not lead to harsh conditions at the core boundaries. These observations are hard to reconcile with simplified simulations of turbulence alone, requiring magnetic fields or other softening agents on small scales Kirk et al. (2009).

The launch of Herschel and the commissioning of SCUBA2 at the JCMT in 2009 ensure that there will be ever more impressive far infrared and (sub)millimeter maps of nearby star-forming regions by 2011 when ALMA begins Early Science.

\section{Acknowledgements}

DJ thanks his collaborators on these projects: H. Kirk, J. Di Francesco, M. Tafalla, J. Jørgensen, S. Basu and all the members of the COMPLETE Team. DJ is supported by a Natural Sciences and Engineering Research Council Discovery Grant.

\section{References}

Evans, N. J., II et al. 2003, PASP, 115, 965

Hatchell, J. \& Fuller, G. A. 2009, A\&A, 482, 855

Johnstone, D., Di Francesco, J., \& Kirk, H. 2004, ApJL, 611, 45

Jørgensen, J. K., Johnstone, D., Kirk, H., \& Myers, P. C. 2007, ApJ, 656, 293

Jørgensen, J. K., Johnstone, D., Kirk, H., Myers, P. C. et al. 2008, ApJ, 683, 822

Kirk, H., Johnstone, D., \& Di Francesco, J. 2006, ApJ, 646, 1009

Kirk, H., Johnstone, D., \& Tafalla, M. 2007, ApJ, 668, 1042

Kirk, H., Johnstone, D., \& Basu, S. 2009, ApJ, 699, 1433

Lombardi, M. \& Alves, J. 2001, A\&A, 377, 1023

Rosolowsky, E. W., Pineda, J. E., Foster, J. B., Borkin, M. A. et al. 2008, ApJS, 175, 509

Swift, J. \& Williams, J. 2008, ApJ, 679, 552

Ward-Thompson, D., André, P., Crutcher, R. et al. 2007, Protostars and Planets V, 33 